\title{
Research Paper: A New and Simple Method for Spinal Cord Injury Induction in Mice
}

\author{
Zahra Zeraatpisheh ${ }^{1,2}$ (1), Esmaeil Mirzaei ${ }^{3,4}$ (), Mohammad Nami ${ }^{1,2}$ (), Hamed Alipour ${ }^{5}$ (1), Somayeh Ghasemian ${ }^{6}$ (1), Hassan Azari ${ }^{7}$, \\ Hadi Aligholi ${ }^{1,2 *}$ (1) \\ 1. Department of Neuroscience, School of Advanced Medical Sciences and Technologies, Shiraz University of Medical Sciences, Shiraz, Iran. \\ 2. Neuroscience Laboratory (Brain, Cognition and Behavior), Department of Neuroscience, School of Advanced Medical Sciences and Technologies, \\ Shiraz University of Medical Sciences, Shiraz, Iran \\ 3. Department of Medical Nanotechnology, School of Advanced Medical Sciences and Technologies, Shiraz University of Medical Sciences, Shiraz, Iran \\ 4. Pharmaceutical Sciences Research Center, Shiraz University of Medical Sciences, Shiraz, Iran \\ 5. Department of Tissue Engineering \& Applied cell Sciences, School of Advanced Medical Sciences and Technologies, Shiraz University of Medical \\ Sciences, Shiraz, Iran. \\ 6. Genetic Laboratory, Shiraz Fertility Center, Shiraz, Iran. \\ 7. Department of Neurosurgery, McKnight Brain Institute, University of Florida, Gainesville, Florida 32611, USA.
}

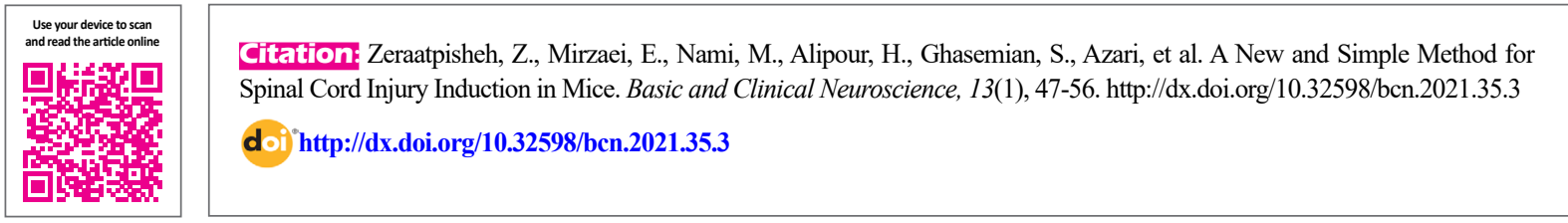

\section{(c) (1) (s)}

Article info:

Received: 28 Oct 2019

First Revision: 05 Mar 2020

Accepted: 12 Jul 2020

Available Online: 01 Jan 2022

Keywords:

Spinal cord injury, Animal models, Mice

\section{A B S T RA C T}

Introduction: Spinal Cord Injury (SCI) is a devastating disease with poor clinical outcomes. Animal models provide great opportunities to expand our horizons in identifying SCI pathophysiological mechanisms and introducing effective treatment strategies. The present study introduces a new murine contusion model.

Methods: A simple, cheap, and reproducible novel instrument was designed, which consisted of a body part, an immobilization piece, and a bar-shaped weight. The injury was inflicted to the spinal cord using an 8-g weight for 5, 10, or 15 minutes after laminectomy at the T9 level in male C57BL/6 mice. Motor function, cavity formation, cell injury, and macrophage infiltration were evaluated 28 days after injury.

Results: The newly designed instrument minimized adverse spinal movement during injury induction. Moreover, no additional devices, such as a stereotaxic apparatus, were required to stabilize the animals during the surgical procedure. Locomotor activity was deteriorated after injury. Furthermore, tissue damage and cell injury were exacerbated by increasing the duration of weight exertion. In addition, macrophage infiltration around the injured tissue was observed 28 days after injury.

Conclusion: This novel apparatus could induce a controllable SCI with a clear cavity formation in mice. No accessory elements are needed, which can be used in future SCI studies.

\section{* Corresponding Author:}

Hadi Aligholi, PhD.

Address: Department of Neuroscience, School of Advanced Medical Sciences and Technologies, Shiraz University of Medical Sciences, Shiraz, Iran. Tel: +98 (71) 32305471

E-mail: aligholi@sums.ac.ir 


\section{Highlights}

- A simple and precise method has been introduced for creating Spinal Cord Injury (SCI) in mice by a novel device.

- The device consists of a body part, an immobilization piece, and a bar-shaped weight.

- Assessment of locomotor activity, tissue damage, and macrophage infiltration confirmed the capability of the new SCI method.

- Reduction of adverse spinal movements and working without any accessory elements are the key points of this new animal model of SCI.

\section{Plain Language Summary}

Spinal Cord Injury (SCI) is a medical problem that can cause the permanent motor and sensory dysfunction. Traffic accidents, falls, and violence are the most frequent causes of SCI, often affecting young people. Patients and even their families may encounter other problems, including reducing life quality, psychological burden, and enormous medical costs. Despite scientific and technological advances, no effective treatment has been found for SCI. Therefore, animal models help study damage mechanisms and evaluate novel treatment strategies. All SCI research centers require an economical and reproducible device without using complex surgical procedures by experienced surgeons to minimize variations in damage to the spinal cord. In this study, a simple, cheap, and reproducible novel instrument for SCI induction is introduced. The instrument consists of various parts, including a body part, an immobilization piece, and a bar-shaped weight. An 8-g weight was used for 5, 10, or 15 minutes to inflict injury to the spinal cord. Behavioral and tissue studies indicated that SCI could be induced in rodents in different severity without other elements. This instrument can be used in future investigations for SCI studies, including tissue engineering, stem cell therapy, and drugs delivery to access effective treatment.

\section{Introduction}

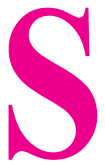

pinal Cord Injury (SCI) is a major medical problem with severe disability and a high mortality rate. Because of the important role of the spinal cord as the connecting pathway for most neural control mechanisms, its injuries cause dysfunction of motor, sensory and autonomic systems (Khan, Havey, Sayers, Patwardhan, \& King, 1999; Yip \& Malaspina, 2012). SCI is created not only by primary mechanical damage but also through secondary mechanisms. Despite all scientific and technological advances, there is still no definitive treatment for this progressive neurological disease. Thus, animal models can be helpful in terms of understanding the molecular pathways involved in secondary damages and evaluating novel treatment strategies (Cheriyan et al., 2014; Onifer, Rabchevsky, \& Scheff, 2007).

Animal models of SCI are classified into transection, contusion, and compression models. Transection is rarely seen clinically, but contusion and compression injuries are more common (McDonough \& Martínez-Cerde- ño, 2012). Transection SCI models include all forms of spinal cord laceration. Two common transection models consist of complete transection, in which the distal part of the whole spinal cord is disconnected from the more proximal parts, and hemisection, in which half of the spinal cord is severed. Complete severance of axons and the formation of a glial scar occur in the transected area (Breyer et al., 2017; Oniferet, al., 2007; Xu, Guénard, Kleitman, \& Bunge, 1995)

In contusion injuries, a weight drop method or a dedicated apparatus such as the New York University, Ohio State University, or Infinite horizon impactor is used (Cheriyan et al., 2014). The severity of the injury depends on the weight or the height from which the impactor tip is dropped. This experimental model results in cavity formation within the spinal cord tissue while the glia limitans remain intact (McDonough \& MartínezCerdeño, 2012). Application of the available devices for SCI induction is limited by their expensive costs and the requirement of complex surgical procedures by experienced surgeons to minimize variations in damage (Farooque, 2000; Kwon, Oxland, \& Tetzlaff, 2002). 
The compression injury can be created by calibrated forceps (McDonough, Monterrubio, Ariza, \& MartínezCerdeño, 2015; Plemel et al., 2008), aneurysm clips (Rivlin \& Tator, 1978), or putting weight directly on to the spinal cord (Wu, Shibuya, Miyamoto, Itano, \& Yamamoto, 2005). Compression is similar to contusion in pathological features and neurological impairment. The severity of an injury can be increased by enhancing compression power; aneurysm clips can apply greater pressure or increase the duration of the spinal cord compression (Cheriyan et al., 2014). Calibrated forceps are a simple, inexpensive, consistent and highly reproducible method. However, recording the amount of force is not possible.

In clip compression, the force velocity and compression extent cannot be recorded (Abdullahi, Annuar, Mohamad, Aziz, \& Sanusi, 2017). In the weight-drop compression model, the spinal cord is only briefly impacted, and thus, it does not reproduce the effect of prolonged cord compression. Considering these limitations, improvement of the current SCI animal models is required.

The present study introduces a feasible method for creating SCI in rodents using a simple, inexpensive, and well-designed novel instrument. For calibration, several factors were evaluated, including quantitative functional outcomes, size of tissue damage, rate of cell injury, and macrophage infiltration.

\section{Methods}

First, a new device was designed and built to induce SCI in rodents. Then to evaluate the effectiveness of our new device, 22 adult male BALB/C mice (26 to $30 \mathrm{~g}$ weight) were used to develop compression SCI. Mice were randomly divided into four groups, including one control (CNT) group, which underwent laminectomy without cord injury, and three SCI groups in which damage was induced with an 8-g weight applied for 5, 10, and $15 \mathrm{~min}$. Subsequently, behavioral and histological assessments were performed. The animals were purchased from the Center of Comparative and Experimental Medicine at the Shiraz University of Medical Sciences in Iran. They were housed in the Animal Lab of the School of Advanced Medical Sciences and Technologies in accordance with standard conditions of ambient temperature $\left(22 \pm 2^{\circ} \mathrm{C}\right), 12: 12 \mathrm{~h}$ light:dark cycle, and free access to water and food. All procedures were done following the institutional guidelines of Shiraz University of Medical Sciences for animal care and use.

\section{SCI apparatus}

As shown in Figure 1, the SCI induction device consists of a body part, an immobilization piece, and a barshaped weight. On each side of the body, a three-legged stand is fixed on a $35 \times 25 \mathrm{~cm}$ long plate to increase the stability of the device at the top of the instrument, and a $15 \times 3.5 \mathrm{~cm}$-long rectangular piece with a hole in the middle. The immobilization piece is similar to the abovementioned rectangle plus four prong-like pieces below. The immobilization piece can be moved up and down in a vertical line up to $2.5 \mathrm{~cm}$. This part of the device is used to fix the spine and prevent its probable vertical and horizontal movements during the SCI procedure. The weight is an 8-g stainless steel metal rod with $27 \mathrm{~cm}$ in length and a $1 \times 2 \mathrm{~mm}$ tip. The tip is curved to fit completely on the surface of the spinal cord. When used, the rod is passed through the holes in the middle of the upper part and the immobilization piece. A $10 \times 32 \mathrm{~cm}$ plate is considered the animal bed, serving the purpose of keeping the animal under the device.

\section{SCI induction}

Before surgery, all surgical tools were sterilized to prevent any possible contamination. Firstly, the animal was weighed and then anesthetized by ketamine $(90 \mathrm{mg} / \mathrm{kg})$ and xylazine $(10 \mathrm{mg} / \mathrm{kg})$. Then, the mouse was placed on a surgical table, sterilized with $70 \%$ alcohol. A heat pad was used to maintain body temperature. To ensure proper anesthesia, the animal was checked for reflexes using the toe pinch. The dorsal side was covered by a surgical drape with an aperture above the surgical area. To prevent ocular damage, eye ointment was applied to the cornea. After shaving the dorsal area around the incision site, the skin was cleaned with Betadine solution, and a longitudinal incision was made using a scalpel blade to expose muscles and vertebral column at the T8-T12.

To keep the incision widely open, the skin was held back with a retractor, and muscles and fascia along the spinal cord were cut. The tissue was detached from the spinous process and posterior lamina of T9. Without any damage or pressure to the spinal cord, connections between the upper and lower vertebrae were slowly drilled, and the T9 posterior lamina was carefully removed with forceps to expose the spinal cord. Subsequently, the mouse was kept on the animal bed and transferred under the SCI device. After that, the immobilization piece was completely fixed on the upper surface of the spine so that the middle hole was placed adjacent to the exposed spinal cord. Then, the weight was passed through the holes in the middle of the upper part and the immobilization 

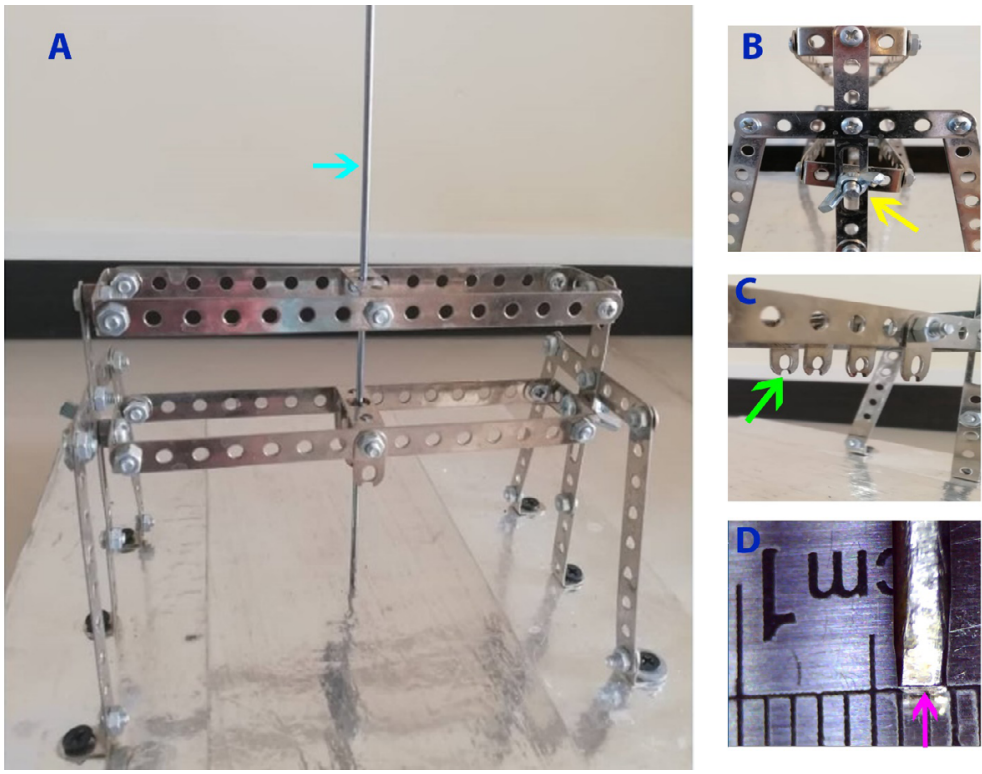

Figure 1. Spinal Cord Injury (SCI) apparatus

NEUR SCIENCE

The device used to create SCI in rodents is illustrated in A. The injury is made by a bar-shaped weight (blue arrow). The immobilization piece (B) can move up and down in a vertical line (yellow arrow). Four prong-like pieces (C, green arrow) are located below the immobilization piece for fixing the spine. The tip of the weight is curved (D, pink arrow), similar to the surface of the spinal cord. The device works without any accessories.

piece and placed on the top of the exposed spinal cord for a defined period (Figure 2). After setting the weight on the spinal cord, sterile saline was applied to regain and keep homeostasis. The muscular layer and skin over the spinal cord were carefully sutured. One milliliter of saline was injected subcutaneously to prevent dehydration. When the animals were awakened, $1.5 \mathrm{mg} / \mathrm{kg}$ of tramadol was given and repeated every 12 hours for 48 hours. In addition, $4 \mathrm{mg} / \mathrm{kg}$ of gentamicin was administrated once a day for 3 days. Mice were separately kept in cages in an environment with appropriate temperature and a 12-h light-dark cycle for 4 weeks after surgery. Mice were weighed on the first day after surgery and then at the end of each week. The bladder was manually
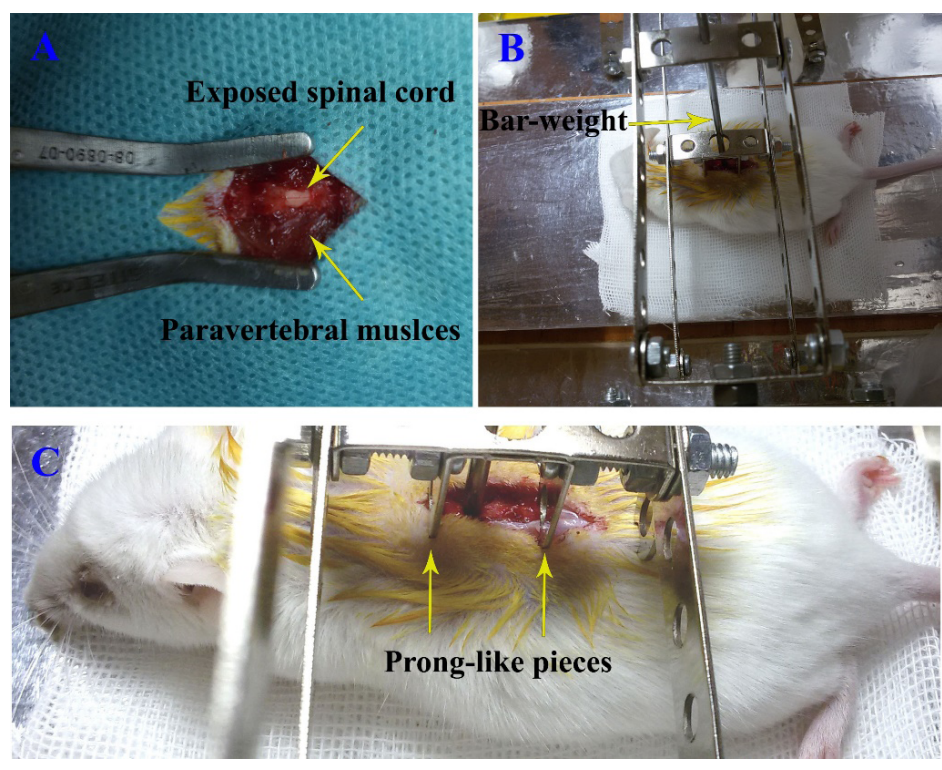

Figure 2. Induction of Spinal Cord Injury (SCI) by the new device

After laminectomy and exposing spinal cord (A), the animal was transferred under the SCI device (B), and the spine was fixed by prong-like pieces of immobilization piece (C). 
A

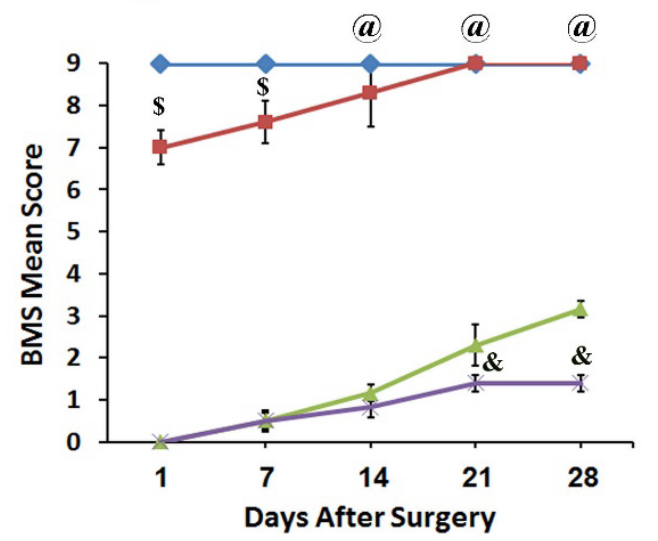

B

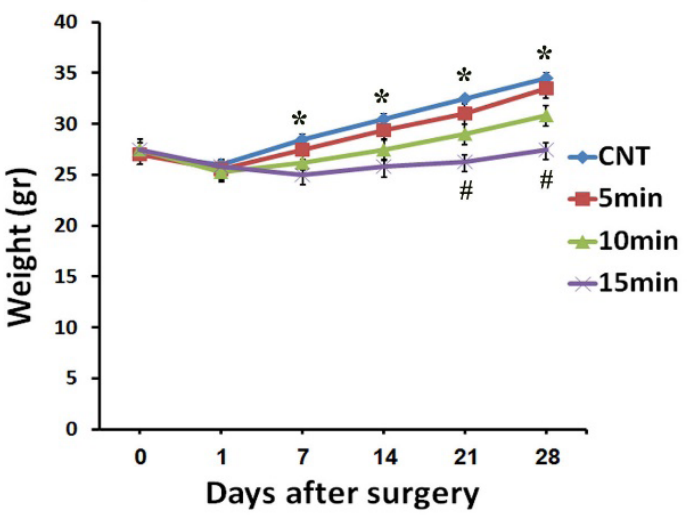

Figure 3. Locomotor behavior and body weight following Spinal Cord Injury (SCI)

NEUR SCIENCE

A) The Basso Mouse Scale (BMS) scores were calculated every week to evaluate the locomotor behavior of mice after SCI. B) The animals' bodyweight was assessed weekly in the different groups.

$\$: \mathrm{P}<0.01$ vs the other groups, @: $\mathrm{P}<0.001$ vs the 10 -min and 15 -min groups, \&: $\mathrm{P}<0.01$ vs the 10 -min group, : $\mathrm{P}<0.05$ vs the 10 -min and 15-min groups, \#: $\mathrm{P}<0.05$ vs the 5 -min and 10-min groups

expressed three times a day until bladder reflex occurred. In the first days, animals were monitored for infection (bloody or cloudy urine), weight loss, and mobility.

Behavioral assessment

We used the Basso Mouse Scale (BMS) locomotor scoring system. It is a sensitive, valid, and reliable preclinical screening tool to evaluate motor function recovery in mice. Its scores range from 0 (complete paralysis) to 9 (completely normal) for behavioral assessment
(Basso et al., 2006). Animals were placed on a flat surface, and hindlimb motor function was scored by two independent, blinded observers one day after surgery and then once a week for 4 weeks.

\section{Tissue preparation}

At the end of the fourth week, the animals were anesthetized and sacrificed by intracardial perfusion of $0.9 \%$ cold normal saline followed by $1 \%$ Paraformaldehyde (PFA) in 0.1 M Phosphate-Buffered Saline (PBS) with
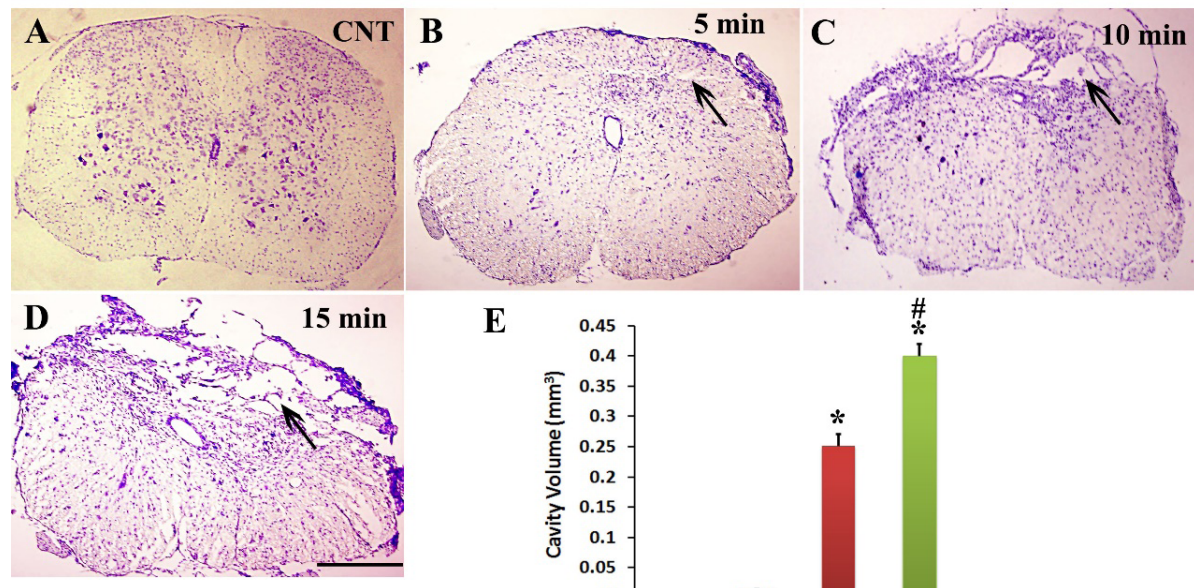

E

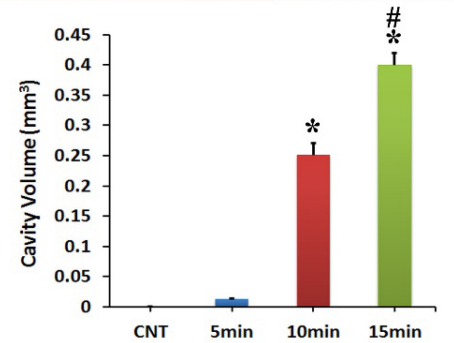

Figure 4. Lesion volume following Spinal Cord Injury (SCI)

By using Nissl-stained sections, the sizes of injury induced by the apparatus in the control (CNT) (A), 5-min (B), 10-min (C), and 15-min (D) groups were calculated, and quantitative results are illustrated in (E).

*: $P<0.001$ vs the 5-min and CNT groups, \#: $\mathrm{P}<0.001$ vs the 10-min group, Scale bar represents $200 \mu \mathrm{m} .=$ 

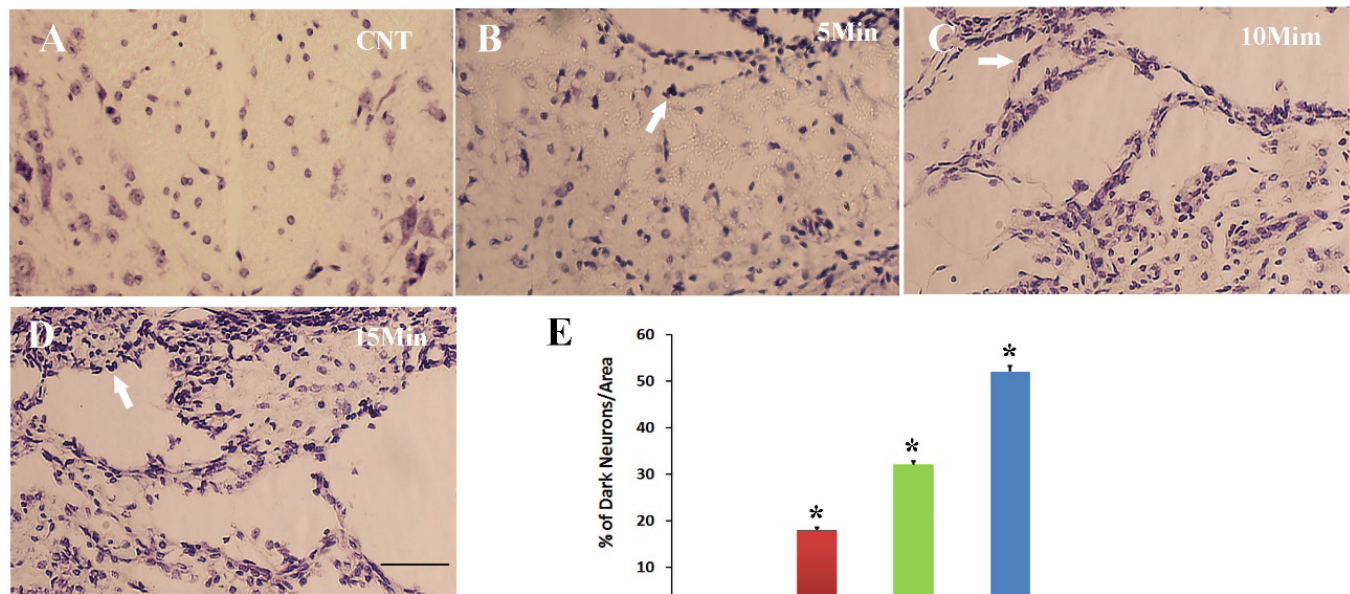

E

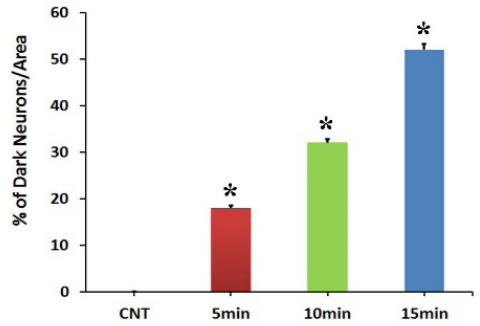

Figure 5. Cell injury following Spinal Cord Injury (SCI)

The percentage of dark cells (white arrow) per area was considered as an index of cell injury in the control (CNT) (A), 5-min (B), 10-min (C), and 15-min (D) groups. The quantitative data are shown in (E).

*: $\mathrm{P}<0.001$ vs the other groups, Scale bar represents $50 \mu \mathrm{m}$

a $\mathrm{pH}$ of 7.4. After opening dorsal skin and breaking the ribs, the spinal column related to the damaged area was removed, excess tissue around it was completely detached and placed in $1 \%$ PFA for $24 \mathrm{~h}$ at $4^{\circ} \mathrm{C}$. After the fixation, the spinal cord was removed from the spinal column by scissors and forceps. For histological studies, the tissues were dehydrated in $70 \%, 80 \%, 96 \%$, and $100 \%$ alcohol, respectively, cleared in xylene and impregnated in paraffin wax.

\section{Nissl staining and measuring the lesion volume}

To assess the volume of the lesion cavity, a series of $8-\mu \mathrm{m}$ sections with an interval of $80 \mu \mathrm{m}$ were prepared and stained with $1 \%$ cresyl violet (Nissl staining) as described elsewhere (Aligholi et al., 2014). The prepared slides were studied under a light microscope, and photos were taken. INFINITY ANALYZE software was used to analyze the photos, and the lesion volume was calculated by the following formula: $0.5 \mathrm{D}(\mathrm{A} 1+\mathrm{An})+\mathrm{D}(\mathrm{A} 2+\mathrm{A}+\ldots$ $+A n-1)$, where $A$ is the area of the cavity and $D$ is the distance between sections (Guo et al., 2009).

\section{Evaluation of cell injury}

The Nissl-stained sections were used to evaluate cell injury. The cells with shrunken features, and corkscrewlike processes, were considered dark cells. The percentage of dark cells per defined area was calculated by the following formula using INFINITY software.

The percentage of dark cells $=$ (the number of dark cells/ total number of cells) $\times 100$ (Ooigawa et al., 2006).

\section{Immunofluorescence assay}

The presence of macrophages around the injury site was investigated by measuring the expression of the CD68 (ED1) as a macrophage marker using immunofluorescence assay. Sections were incubated overnight at $4^{\circ} \mathrm{C}$ with rabbit anti-CD68 primary antibody $(1 / 200$, Abcam, Germany). Goat anti-rabbit secondary antibodies (1/600, FITC, Abcam, Germany) were applied for 1 $\mathrm{h}$ at room temperature. Slides were visualized using an Olympus microscope, and pictures were taken. Immunofluorescence was quantified by ImageJ software (Jafarian et al., 2019).

\section{Statistical analyses}

All data are presented as Mean \pm Standard Error of the mean. Statistical analyses were calculated using SPSS software, v. 23, Chicago, USA. For assessing changes over different groups, we used 1-way ANOVA followed by a pair-wise comparison of the groups using post hoc testing with LSD correction. A repeated measure ANOVA with a Greenhouse-Geisser correction was used to assess changes over time. In all analyses, the significance level for the overall group effect and individual pair-wise comparisons was set as $\mathrm{P}<0.05$.

\section{Results}

\section{Effect of SCI on locomotor behavior}

As illustrated in Figure 3A, a repeated-measures ANOVA with a Greenhouse-Geisser correction indicated that 

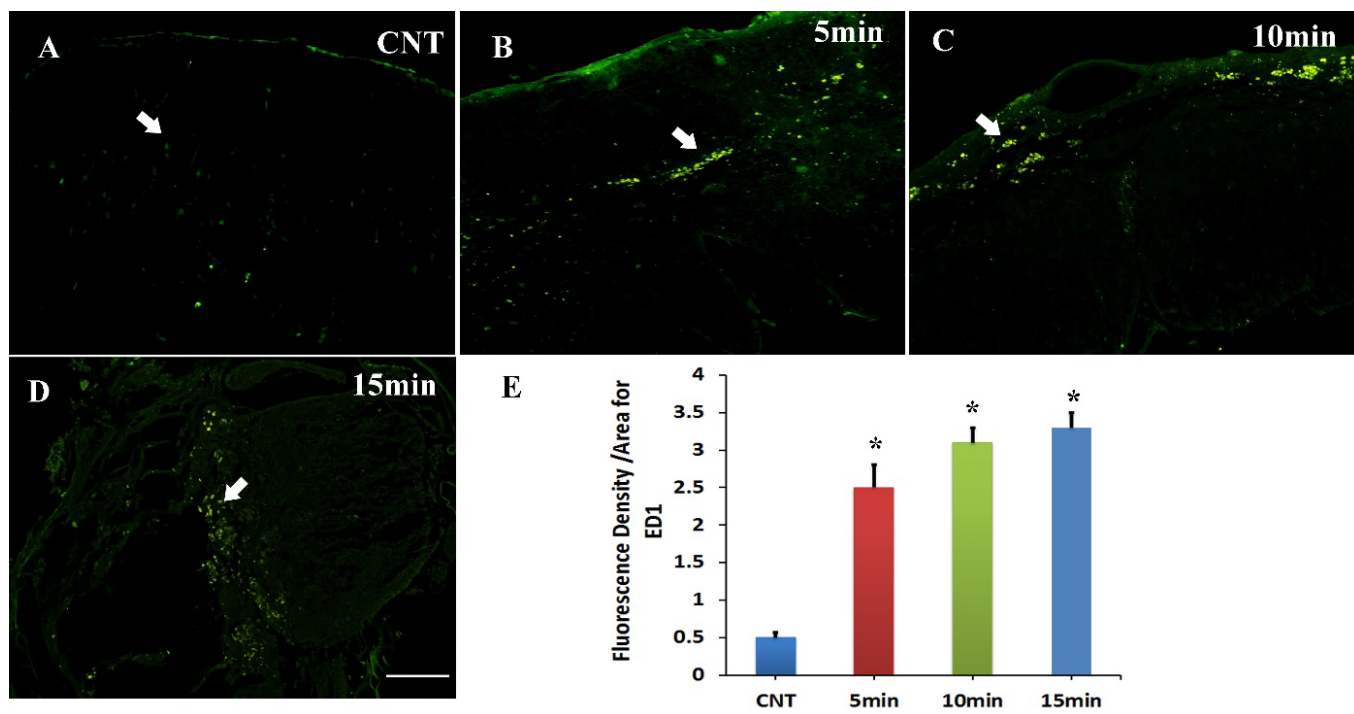

NEUR:SCIENCE

Figure 6. Macrophage infiltration following Spinal Cord Injury (SCI)

The percentage of ED1-positive cells (white arrow) per area was considered as an index of macrophage infiltration in the control (CNT) (A), 5-min (B), 10-min (C), and 15-min (D) groups. (E) indicates the quantitative data.

*: P<0.001 vs the CNT group, Scale bar represents $100 \mu \mathrm{m}$.

BMS mean score differed significantly between time points $\left.\left.\left(\mathrm{F}_{7,44}\right) 11.8\right)=11 ; \mathrm{P}<0.01\right)$. Placing the weight on the spinal cord for 5 minutes could cause mild hindlimb paralysis at day 1 post-injury. BMS mean score in this group was decreased to $7 \pm 0.4$ on day 1 and recovered to $9 \pm 0$ on day 28 . BMS score in the 5-min group was significantly higher on days $1,7,14,21$, and 28 post-injury than that of 10 - and 15 -min groups $(\mathrm{P}<0.01)$. On the other hand, complete hindlimb paralysis was achieved by placing the weight on the spinal cord for 10 and 15 min on day 1 post-injury. The recovery process was accelerated in the 10-min group from day 7 post-injury so that the BMS score of animals in the 10-min group was significantly different from that of the 15-min group on days 21 and 28 post-injury $(\mathrm{P}<0.01)$.

\section{Effect of SCI on body weight}

The body weights of the mice were also evaluated at days $1,7,14,21$, and 28 post-injury $\left(\mathrm{F}_{6,36}=14, \mathrm{P}<0.05\right)$. One day after surgery, all mice showed weight loss, which continued during the first week after surgery in the 15-min group. After that, the weight-gaining process was observed in all groups until 28 days after surgery. However, the body weights of animals in the 5- and 10min groups were considerably higher than that of animals in the 15 -min group on days 21 and 28 post-injury $(\mathrm{P}<0.05$; Figure $3 \mathrm{~B})$.

\section{Effect of SCI on lesion volume}

The size of the cavity created by SCI was measured using Nissl-stained sections. The results showed that this parameter increased as the time of SCI induction increased (Figure 4). The size of cavity in the spinal cord of animals in 10-min group $(0.25 \pm 0.02)$ was significantly more than that of animals in the 5 min group $(0.013 \pm 0.0014, \mathrm{P}<0.001)$. In addition, the size of injury in the 15 -min group $(0.4 \pm 0.02)$ was considerably larger than that of the 5- and 10-min groups $(\mathrm{P}<0.001)$.

\section{Effect of SCI on cell injury}

The percentage of dark neurons as an index of cell injury was evaluated by Nissl staining (Figure 5). The results showed that the number of dark neurons around the injury site in the 10 -min group $(32 \pm 0.8)$ was significantly more compared to the 5-min group (18.26 \pm 0.6 , $\mathrm{P}<0.001)$. Moreover, this index in the 15 -min group $(52 \pm 1.2)$ significantly increased compared to the 5-min and 10-min groups $(\mathrm{P}<0.001)$. The percentages of dark cells in the 5-min, 10-min, and 15-min groups were remarkably higher than those of the CNT group $(\mathrm{P}<0.001)$.

\section{Effect of SCI on macrophage infiltration}

The distribution of ED1 (macrophage marker) was studied by immunofluorescence assay. The results revealed that the distribution of macrophages was signifi- 
cantly higher in the 5-min, $10 \mathrm{~min}$, and 15 min groups than those of the CNT group $(\mathrm{P}<0.001)$. In addition, macrophage infiltration showed an increasing trend from the 5-min group to the 15 min group; however, there was no statistical significance among the groups (Figure 6).

\section{Discussion}

The present study introduced a novel, simple, reproducible, and economical method to produce a mice model of SCI. The efficiency of the method was confirmed by the worsening of locomotor activity scores, increasing tissue damage, and cell injury indices after an increase in injury induction time.

Different models and devices have already been introduced for SCI induction (McDonough \& Martínez-Cerdeño, 2012; Plemel et al., 2008; Rivlin \& Tator, 1978). One of the most commonly used models is the weight-drop technique established by Allen in 1911 (Allen, 1911). After that, efforts were made to create more precise, controllable models that led to the creation of some complex and expensive devices (Deep et al., 2015). Here, we designed and introduced a simple instrument that can be made easily. One of the advantages of this new device is that one can induce SCI without additional devices, like the stereotaxic apparatus. In this sense, the immobilization part of the device helps us to induce SCI with the least adverse spinal movement. Moreover, the induction time and the weight can be changed to create SCI models with different intensities. Furthermore, the device is applicable for induction of SCI in other rodents like rats.

This model of SCI affects the motor function of the hindlimb and the function of the urinary system. After a manual massage of the bladder, the bladder reflex generally returned during the first week after the injury. Using analgesic drugs in the first few days after SCI helps the animal to tolerate pain and facilitate seeking for water and food. The behavioral assessment revealed that hindlimb motor dysfunction correlated with the compression time. In other words, BMS scores reduced when compression time increased. Based on the results of the BMS test, 5 min compression created a weak SCI that was completely recovered after 28 days. However, $10 \mathrm{~min}$ and 15 min compression created a moderate to severe SCI, respectively, which remained for 28 days.

Whole-body weight as a general indication of health was measured in this study. We observed weight loss that usually occurs after SCI (Landry, Frenette, \& Guertin, 2004). Almost all mice had 1-2 g body weight loss one day after injury due to the direct effect of surgery. In the 5-min group, mice compensated for this weight loss quickly during the first week after injury, depending on the injury and consuming enough water and food. In the 10-min group, weight gaining was slower, but the mice compensated for their weight loss around the second week and then followed the natural process of weight gaining. Although the weight gaining was very slow in the 15-min group, there was no excess weight loss after the first week after the injury.

The formation of a cavity after SCI is one of the main pathological features that occurs following secondary injury (Ahuja et al., 2017; Chen et al., 2016; Osaka et al., 2010). During the first hours after primary SCI, the secondary injury starts through several mechanisms, including an increase in intracellular calcium ion, activation of proteases, activation of autophagy, secretion of inflammatory elements, activation of microglia, and migration of macrophages and astrogliosis. Although the first aim of these mechanisms is supporting the damaged tissue, they act as a double-edged sword. After activation of the secondary mechanisms and elimination of the damaged tissue, a cavity is formed during the early weeks following SCI. Thus, the formation of a cavity is one of the necessities in properly establishing animal models of SCI (Deep et al., 2015; Sroga, Jones, Kigerl, McGaughy, \& Popovich, 2003; Surey, Berry, Logan, Bicknell, \& Ahmed, 2014). In this study, we observed cavity formation in all three groups. The cavity was very small in the 5-min group, but by increasing the induction time, the cavity became larger so that they were more obvious in the 10- and 15-min groups. It is worth pointing out that cavity formation in animal models of SCI is an interesting option for scientists' working on neuro-regeneration using tissue engineering.

In the present study, macrophage infiltration was evaluated after SCI. In this sense, the presence of macrophages around the damaged tissue was observed 4 weeks after SCI. Spinal cord injury is associated with macrophage activation; however, the effect of this phenomenon has remained controversial. Like previous reports, the activation of these cells was maximum between 3 and 7 days postinjury (Carlson, Parrish, Springer, Doty, \& Dossett, 1998; Zhang, Krebs, \& Guth, 1997), but Wu et al. demonstrated that they were observed at 4 weeks and reduced around 12 weeks after injury in rats (Wu, et al., 2005). Thus by using our model of SCI, the activation of macrophages can be evaluated up to 4 weeks post-injury in mice. 


\section{Conclusion}

In conclusion, using this new and simple apparatus, a controllable SCI can be induced in rodents without accessory elements. The creation of compression injury with a clear cavity can be helpful in future tissue engineering-based investigations for SCI.

\section{Ethical Considerations}

\section{Compliance with ethical guidelines}

There were no ethical considerations to be considered in this research

\section{Funding}

The present study was supported by Shiraz University of Medical Sciences and Health Services (1396-01-74-14199).

\section{Authors' contributions}

Conceptualization: Hadi Aligholi, Esmaeil Mirzaei, Mohammad Nami, and Hassan Azari; Methodology: Hadi Aligholi, Esmaeil Mirzaei, Mohammad Nami, and Hassan Azari; Investigation: Zahra Zeraatpisheh, Hamed Alipour, and Somayeh Ghasemian; Writing the original draft: Zahra Zeraatpisheh, Hamed Alipour, and Hadi Aligholi; Writing, review, and editing: all authors; Funding acquisition: Hadi Aligholi; Supervision: Hadi Aligholi, Esmaeil Mirzaei, Mohammad Nami, and Hassan Azari.

\section{Conflict of interest}

The authors declare that they have no conflict of interest.

\section{Acknowledgments}

Authors appreciate the from Shiraz University of Medical Sciences fot their support.

\section{References}

Abdullahi, D., Annuar, A. A., Mohamad, M., Aziz, I., \& Sanusi, J. (2017). Experimental spinal cord trauma: A review of mechanically induced spinal cord injury in rat models. Reviews in the Neurosciences, 28(1), 15-20. [DOI:10.1515/revneuro-2016-0050] [PMID]

Ahuja, C. S., Wilson, J. R., Nori, S., Kotter, M. R., Druschel, C., Curt, A., et al. (2017). Traumatic spinal cord injury. Nature Reviews. Disease Primers, 3, 17018. [DOI:10.1038/nrdp.2017.18] [PMID]
Aligholi, H., Hassanzadeh, G., Azari, H., Rezayat, S. M., Mehr S. E., Akbari, M., et al. (2014). A new and safe method for stereotactically harvesting neural stem/progenitor cells from the adult rat subventricular zone. Journal of Neuroscience Methods, 225, 81-9. [DOI:10.1016/j.jneumeth.2013.12.008] [PMID]

Allen, A. R. (1911). Surgery of experimental lesion of spinal cord equivalent to crush injury of fracture dislocation of spinal column: A preliminary report. Journal of the American Medical Association, 57(11), 878-80. [DOI:10.1001/ jama.1911.04260090100008]

Basso, D. M., Fisher, L. C., Anderson, A. J., Jakeman, L. B., Mctigue, D. M., \& Popovich, P. G. (2006). Basso Mouse Scale for locomotion detects differences in recovery after spinal cord injury in five common mouse strains. Journal of neurotrauma 23(5), 635-59. [DOI:10.1089/neu.2006.23.635] [PMID]

Breyer, B. N., Fandel, T. M., Alwaal, A., Osterberg, E. C., Shindel, A. W., Lin, G., et al. (2017). Comparison of spinal cord contusion and transection: Functional and histological changes in the rat urinary bladder. BJU International, 119(2), 333-41. [DOI:10.1111/bju.13591] [PMID]

Carlson, S. L., Parrish, M. E., Springer, J. E., Doty, K., \& Dossett, L. (1998). Acute inflammatory response in spinal cord following impact injury. Experimental Neurology, 151(1), 77-88. [DOI:10.1006/exnr.1998.6785] [PMID]

Chen, K., Liu, J., Assinck, P., Bhatnagar, T., Streijger, F., Zhu, Q et al. (2016). Differential histopathological and behavioral outcomes eight weeks after rat spinal cord injury by contusion, dislocation, and distraction mechanisms. Journal of Neurotrauma, 33(18), 1667-84. [DOI:10.1089/neu.2015.4218] [PMID] [PMCID]

Cheriyan, T., Ryan, D., Weinreb, J., Cheriyan, J., Paul, J., Lafage, V., et al. (2014). Spinal cord injury models: A review. Spina Cord, 52(8), 588-95. [DOI:10.1038/sc.2014.91] [PMID]

Deep, A., Adeeb, N., Hose, N., Rezaei, M., Fard, S. A., Tubbs, R. S., et al. (2015). Mouse models of spinal cord injury and stem cell transplantation. Translational Research in Anatomy, 1 2-10. [DOI:10.1016/j.tria.2015.10.001]

Farooque, M. (2000). Spinal cord compression injury in the mouse: Presentation of a model including assessment of motor dysfunction. Acta Neuropathologica, 100(1), 13-22. [DOI:10.1007/s004010051187] [PMID]

Guo, J., Leung, K. K. G., Su, H., Yuan, Q., Wang, L., Chu, T. H., et al. (2009). Self-assembling peptide nanofiber scaffold promotes the reconstruction of acutely injured brain. Nanomedicine: Nanotechnology, Biology and Medicine, 5(3), 345-51. [DOI:10.1016/j.nano.2008.12.001] [PMID]

Jafarian, M., Mousavi, S. M. M., Alipour, F., Aligholi, H., Noorbakhsh, F., Ghadipasha, M., et al. (2019). Cell injury and receptor expression in the epileptic human amygdala. Neurobiology of Disease, 124, 416-27. [DOI:10.1016/j.nbd.2018.12.017] [PMID]

Khan, T., Havey, R. M., Sayers, S. T., Patwardhan, A., \& King, W. W. (1999). Animal models of spinal cord contusion injuries. Comparative Medicine, 49(2), 161-72. https:/ / www.ingentaconnect.com/content/aalas/cm/1999/00000049/00000002/ $\operatorname{art00008}$

Kwon, B. K., Oxland, T. R., \& Tetzlaff, W. (2002). Animal models used in spinal cord regeneration research. Spine, 27(14), 1504 10. [DOI:10.1097/00007632-200207150-00005] [PMID] 
Landry, E., Frenette, J., \& Guertin, P. A. (2004). Body weight, limb size, and muscular properties of early paraplegic mice. Journal of Neurotrauma, 21(8), 1008-16. [DOI:10.1089/0897715041651060] [PMID]

McDonough, A., \& Martínez-Cerdeño, V. (2012). Endogenous proliferation after spinal cord injury in animal models. Stem Cells International, 2012, 387513. [DOI:10.1155/2012/387513] [PMID] [PMCID]

McDonough, A., Monterrubio, A., Ariza, J., \& Martínez-Cerdeño, V. (2015). Calibrated forceps model of spinal cord compression injury. Journal of Visualized Experiments, (98), 52318. [DOI:10.3791/52318] [PMID] [PMCID]

Onifer, S. M., Rabchevsky, A. G., \& Scheff, S. W. (2007). Rat models of traumatic spinal cord injury to assess motor recovery. ILAR Journal, 48(4), 385-95. [DOI:10.1093/ilar.48.4.385] [PMID]

Ooigawa, H., Nawashiro, H., Fukui, S., Otani, N., Osumi, A., Toyooka, T., et al. (2006). The fate of Nissl-stained dark neurons following traumatic brain injury in rats: Difference between neocortex and hippocampus regarding survival rate. Acta Neuropathologica, 112(4), 471-81. [DOI:10.1007/s00401006-0108-2] [PMID]

Osaka, M., Honmou, O., Murakami, T., Nonaka, T., Houkin, K. Hamada, H., et al. (2010). Intravenous administration of mesenchymal stem cells derived from bone marrow after contusive spinal cord injury improves functional outcome. Brain Research 1343, 226-35. [DOI:10.1016/j.brainres.2010.05.011] [PMID]

Plemel, J. R., Duncan, G., Chen, K. W. K., Shannon, C., Park, S., Sparling, J. S., et al. (2008). A graded forceps crush spinal cord injury model in mice. Journal of Neurotrauma, 25(4), 350-70. [DOI:10.1089/neu.2007.0426] [PMID]

Rivlin, A., \& Tator, C. (1978). Effect of duration of acute spinal cord compression in a new acute cord injury model in the rat. Surgical Neurology, 10(1), 38-43. [PMID]

Sroga, J. M., Jones, T. B., Kigerl, K. A., McGaughy, V. M., \& Popovich, P. G. (2003). Rats and mice exhibit distinct inflammatory reactions after spinal cord injury. Journal of Comparative Neurology, 462(2), 223-40. [DOI:10.1002/cne.10736] [PMID]

Surey, S., Berry, M., Logan, A., Bicknell, R., \& Ahmed, Z. (2014). Differential cavitation, angiogenesis and wound-healing responses in injured mouse and rat spinal cords. Neuroscience, 275, 62-80. [DOI:10.1016/j.neuroscience.2014.06.003] [PMID]

Wu, D., Miyamoto, O., Shibuya, S., Okada, M., Igawa, H., Janjua, N. A., et al. (2005). Different expression of macrophages and microglia in rat spinal cord contusion injury model at morphological and regional levels. Acta Medica Okayama, 59(4), 121-7. [DOI:10.18926/AMO/31950] [PMID]

Wu, D., Shibuya, S., Miyamoto, O., Itano, T., \& Yamamoto, T. (2005). Increase of NG2-positive cells associated with radial glia following traumatic spinal cord injury in adult rats. Journal of Neurocytology, 34(6), 459-69. [DOI:10.1007/s11068 006-8998-4] [PMID]

Xu, X. M., Guénard, V., Kleitman, N., \& Bunge, M. B. (1995). Axonal regeneration into Schwann cell-seeded guidance channels grafted into transected adult rat spinal cord. Journal of Comparative Neurology, 351(1), 145-60. [DOI:10.1002/ cne.903510113] [PMID]
Yip, P. K., \& Malaspina, A. (2012). Spinal cord trauma and the molecular point of no return. Molecular Neurodegeneration, 7, 6 . [DOI:10.1186/1750-1326-7-6] [PMID] [PMCID]

Zhang, Z., Krebs, C. J., \& Guth, L. (1997). Experimental analysis of progressive necrosis after spinal cord trauma in the rat: Etiological role of the inflammatory response. Experimental Neurology, 143(1), 141-52. [DOI:10.1006/exnr.1996.6355] [PMID] 